Article

\title{
Time-Dependent Probabilistic Model for Hierarchical Structure in Failure Mode and Effect Analysis
}

\author{
Hyeon-ae Jang ${ }^{1}$ and Seungsik Min ${ }^{2, * \mathbb{D}}$ \\ Research \& Business Foundation, Dong-A University, Busan 49315, Korea; jha3562@gmail.com \\ 2 Department of Natural Science, Korea Naval Academy, Changwon 51704, Korea \\ * Correspondence: fieldsmin@gmail.com; Tel.: +82-55-907-5238
}

Received: 1 August 2019; Accepted: 4 October 2019; Published: 11 October 2019

\begin{abstract}
Failure mode and effect analysis (FMEA) is a structured technique for identifying risks that may occur during a given stage of a system's life cycle. However, the use of the risk priority number (RPN) in traditional FMEA results in difficulties with regard to quantification of the degree of risk in the hierarchical failure structure. This study proposes the use of a hierarchical time-dependent FMEA approach to overcome the limitations encountered during the implementation of traditional FMEA approaches. In place of the RPN, a probabilistic loss model is developed under a hierarchical structure considering the elapsed time from the failure-cause (FC) to the system failure. By assuming exponential and case functions for each occurrence and detection time instant, the expected loss corresponding to each FC can be evaluated. As a result of the practical application of the time-dependent probabilistic model through the numerical example, we could reasonably evaluate the risk from the cause of failure in the hierarchical structure in terms of economic loss.
\end{abstract}

Keywords: FMEA; HTD-FMEA; hierarchical structure; time-dependent probabilistic loss model; expected loss; risk evaluation

\section{Introduction}

For assuring the safety and reliability of a system, engineers identify all possible failures and their corresponding failure-causes (FCs) and take actions to reduce the occurrence of FCs or mitigate the loss due to their consequences. Failure mode and effect analysis (FMEA) is a structured method used for defining, identifying, and mitigating known and/or potential causes of failures, problems, errors, etc. in systems, designs, processes, and services prior to them being made accessible to customers [1]. Since its introduction as an analysis tool for failure mitigation, FMEA has been extensively used in a wide range of industrial applications, including, but not limited to, automotive, semiconductor design, aerospace and aviation, healthcare, and steel manufacturing applications [2-5]. In traditional FMEA approaches, the risk associated with an FC is usually evaluated using the risk priority number (RPN), which corresponds to the mathematical product of the occurrence (O), severity (S), and detection (D) of an FC. However, this traditional FMEA risk-evaluation approach has often been extensively criticized in extant literature for a variety of reasons [6]. The major shortcomings of the traditional RPN-based approach are as follows: (i) The relative importance among $\mathrm{O}, \mathrm{S}$, and D is not considered [7-10]; (ii) the three risk factors are difficult to precisely evaluate [4,11-17]; (iii) interdependencies among various failure modes and effects are not considered [1,5,18,19]; (iv) the method depends on experts' intuition and experience rather than the scientific method to estimate the three risk components [20,21]; and (v) there is no consideration of possible hierarchical relationships among failures [6,22,23].

Many researchers have attempted to improve the RPN-based risk-evaluation method of FMEA. Some researchers have considered facilitating the assessment of the three risk-evaluation factors- $\mathrm{O}, \mathrm{S}$, and D—by adopting fuzzy logic-based approaches $[4,5,7,10,12,19,24-26]$ or fuzzy rule-based Bayesian 
reasoning approaches [27]. Other researchers $[9,28]$ employed both grey and fuzzy theories in FMEA. Geum et al. [2] proposed a systematic approach to evaluate potential failures using a service-specific FMEA and grey relational analysis. Jih [29] evaluated the structure of hierarchy and interdependence of corrective action, then calculated the weight of corrective action through the analytic network process (ANP). Hu et al. [30] employed both FMEA and fuzzy analytic hierarchy process (FAHP) techniques to recognize risk-evaluation criteria. Zammori and Gabbrielli [31] tried to identify possible interactions among the principal causes of failure by integrating ANP and a multi-criteria decision-making technique. Some researchers have proposed an integrated FMEA technique combining the various methods, i.e., the decision-making method [18,20]; ordered weighted geometric averaging (OWGA) [8]; the linguistic distribution assessment method [16]; Dempster-Shafer theory (DST) [15]; fault-tree analysis (FTA) [32]; robust data-envelopment analysis (RDEA) [21]; and failure modes, mechanisms, and effect analysis (FMMEA) [17]. Parracho Sant'Anna [14] suggested a method based on treating the numerical initial measurements as estimates of the location parameters of probability distributions, which enables objective consideration of the uncertainty inherent in such measurements and computation of the probabilities of each potential failure being the most important according to each criterion. Kim \& Zuo [1] presented a general model to explain the functional relationship among the three following factors: Severity, probability of occurrence, and detection. Based on the functional relationship, they calculated the risk of failure mode according to the conditional probability of three factors. Some researchers have proposed cost-based FMEA technology. Braglia [33] developed a new tool for reliability and FMEA by integrating the conventional aspects of the popular failure mode and criticality analysis (FMECA) procedure with economic considerations. Here, FMECA was approached as a multi-criteria decision-making technique that integrates four different factors, as follows: The chance of failure, the chance of non-detection, the severity, and the expected cost. Based on Braglia's [33] study, Carmignani [34] suggested a new method, priority-cost FMECA, that allows for the calculation of a new RPN and the introduction of the concept of profitability, taking the corrective action cost into consideration. Rhee and Ishii [11] introduced a new methodology, life cost-based FMEA, that measures risk in terms of cost. Life cost-based FMEA is useful to compare and select design alternatives that can reduce the overall life-cycle cost of a particular system. Von Ahsen [13] developed an approach to improve FMEA from an economic perspective by considering the estimated cost of external faults in the evaluation of the severity of failures. Dong [12] proposed the use of a FMEA tool based on fuzzy utility cost estimation to overcome the limitations of conventional FMEA techniques. The utility theory accounted for the nonlinear relationship between the cost incurred in the event of a failure and used ordinal ranking. Kwon et al. [22] suggested the expected loss model, in which failure was dependent on time and the system was periodically monitored to prevent failures during its mission period. The loss due to each failure mode was assumed to depend on the remaining mission period of the system.

Some researchers have accounted for time or a hierarchical structure. For instance, Jang et al. [35] assumed an exponential probability distribution for the evaluation of occurrence and detection times for failures and their corresponding causes, and the expected severity was derived for each FC. A new risk metric (REM) was defined as the product of the FC occurrence rate and the expected severity of the corresponding failure. In another study by Jang et al. [23], both time and a two-level hierarchical structure were employed to evaluate risks of the failure mode and its corresponding cause. Assuming the severity of failure depends on the length of the delayed time for corrective action, a severity model was developed. Using the expected severity, a risk priority metric was defined. Regarding the hierarchical structure, Wada [36] introduced the failure mode factors and effects analysis (FMFEA) technique that allowed hierarchical representations of causes and ripple effects for the failure mode. Jang et al. [37] tried to systematically identify the root causes of failure modes based on a their proposed FMEA sheet and scientifically evaluated the relevant risk priorities for hierarchical FCs. Peeters et al. [32] presented the failures hierarchically through FTA and conducted 
risk assessment through FMEA. All critical risk assessments were performed from the system level to the component level.

To the best of our knowledge, there is no study that simultaneously considers the hierarchical failure structure and the elapsed time after a failure in the FMEA. This paper is intended to develop a hierarchical time-dependent FMEA (HTD-FMEA). If the occurrence of a specific failure is represented in a hierarchical structure, the system (top-level) failure will be caused by at least one of its FCs (bottom level). It will take time for the system failure to occur after the FCs, and the elapsed time from each FC to the system failure will be different from the others. Moreover, the system failure can lead to a serious accident, such as product failure or leakage of a hazardous substance, and the loss due to such an accident will vary depending on the detection time. If the FC is detected before the system failure occurs, only the constant cost of repairing the FC will be required. However, if the FC is detected after its actual occurrence, then the loss may become significantly larger as the undetected time duration of the failure effect becomes larger [23,35].

In this study, we evaluate the risk of failure by quantifying the loss as a function depending on the detection time and the system failure time in the hierarchical system. Assuming the probability density function of the failure occurrence to be exponential and that of the detection to be a case function, the expected value of loss can be evaluated for each FC. We presume that quantitative data are available for risk evaluation so as to focus on explaining our model (time-dependent probabilistic model for hierarchical systems) theoretically and logically. This paper is organized as follows: In Section 2, the background and purpose of this study are explained. Section 3 describes the time-dependent probabilistic model for the hierarchical structure. In Section 4 , an example is provided to verify the effectiveness of HTD-FMEA. Section 5 concludes the paper.

\section{Background}

To ensure the safety and reliability of a system, engineers identify all independent failures and take action to reduce the occurrences of failure or mitigate the severity of their consequences $[1,20,38]$. To prevent failure, it is necessary to systematically analyze the FC and take measures to eliminate the root causes. The FTA as a deductive methodology is a widely used technique to describe the causes in a hierarchical way, but it does not possess a feature to easily assess the risk of failure [32,39]. However, the FMEA does not have a feature to analyze failures hierarchically, but it possesses a feature to focus the analysis on the most critical FC. Some researchers have tried to supplement the shortcoming of FMEA based FTA [23,32,36,37,39]. Peeters et al. [32] performed a case study of failure analysis of an additive manufacturing system for metal printing, the Metal FAB1 of Additive Industries (AI), by combining FTA and FMEA. In their study, failure analysis was performed at the system, function, and component levels. Figure 1 shows a key part of the resulting fault tree at the functional level; to simplify the diagram, some branches are not depicted. The functional failure mode is the "Powder layer deposit failure" at the $0^{\text {th }}$ level (system failure, SF). There are 14 intermediate events (subsystem failures, SSFs), marked with a white box, and 9 component failure modes (FCs), marked with a blue circle. Subsequently, FMEA is performed to risk prioritize the identified component failure modes by means of the RPN $(=\mathrm{O} \times \mathrm{S} \times \mathrm{D})$. It seems logical to analyze the failures hierarchically and determine the risk priorities for the component failure modes (i.e., FCs). This is because, to prevent the occurrence of the functional failure mode, the critical component failure mode must be removed first. 


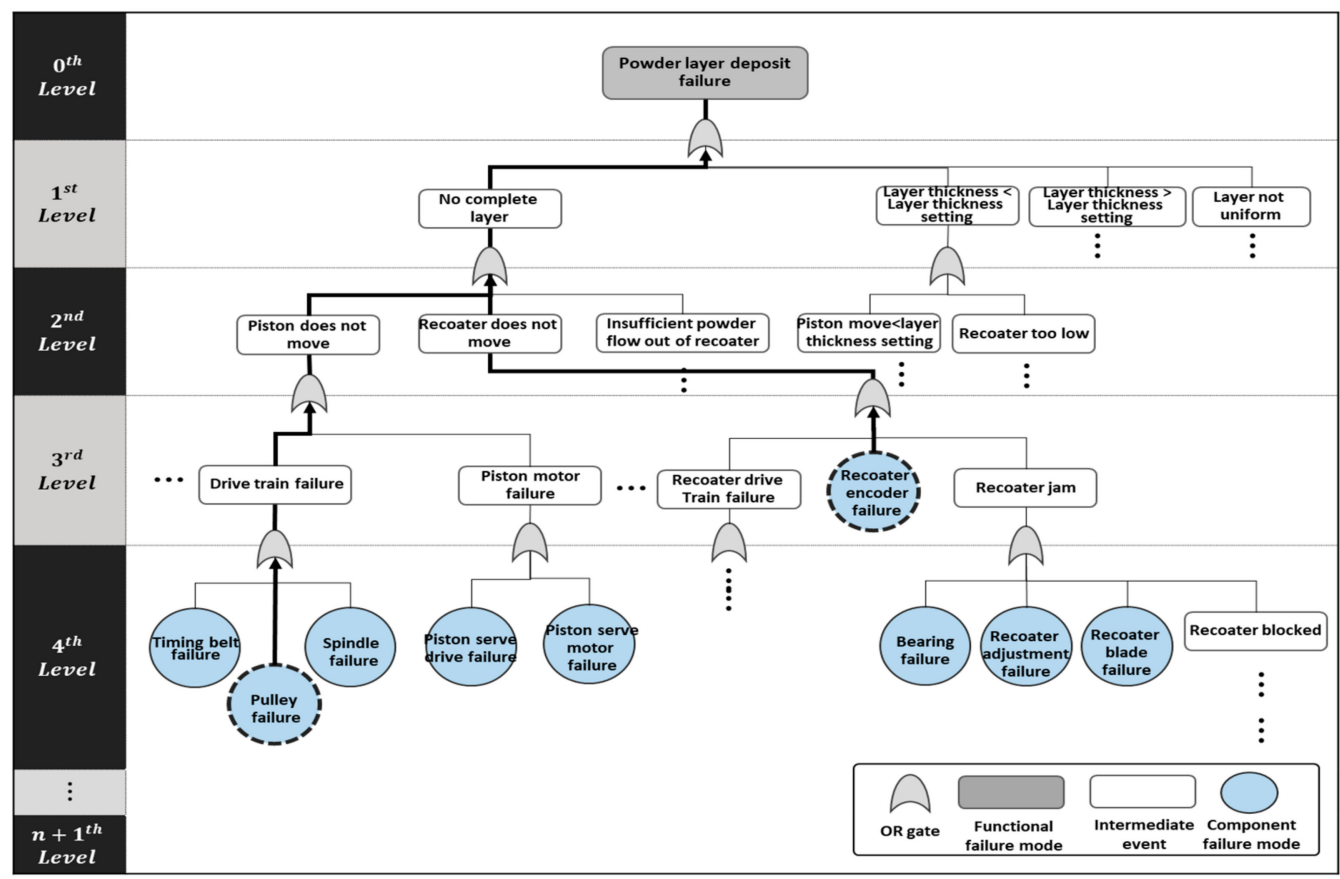

Figure 1. Functional level fault tree analysis (FTA) (partial), adopted from [32].

In Figure 1, the occurrence of "Pulley failure" or "Recoater encoder failure" eventually leads to "Powder layer deposit failure" along each failure path. These two failure paths can be described with the random occurrence and detection time of each failure, as shown in Figure 2. For Case A, the FC (i.e., "Recoater encoder failure") was detected long after the SF (i.e., "Power layer deposit") occurred. In this case, the loss from the failure may be expected to include the cost $\theta$ of the failure effect, along with the constant cost $\alpha$ and $\beta$ for repairing the FC and SF, respectively. On the contrary, in Case B, the FC (i.e., "Pulley failure") was detected before the SF occurred; in this case, only the constant repair cost $\alpha$ is expected.

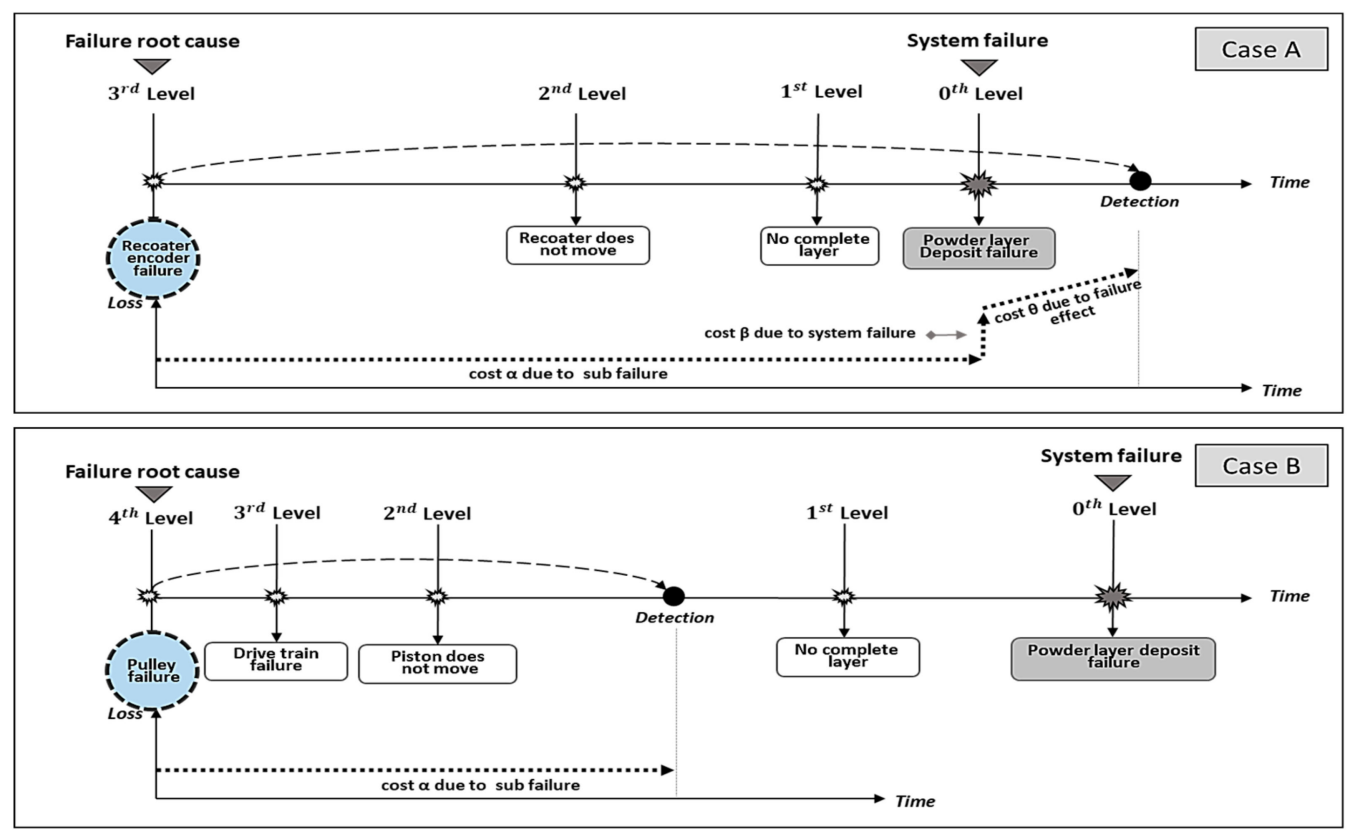

Figure 2. Loss depending on the detectable time in hierarchical failure occurrence. 
If these failures occur as a result of cause and effect in a hierarchical structure, all failures will occur sequentially from the FC. Furthermore, in general, the FC can take an extremely short time or a fairly long time before it is detected. If any FC is found before the SF, only the foreseeable cost for repair will be expended. However, after the SF, the repair cost, as well as the opportunity cost of handling the failure effects, will be borne.

\section{Hierarchical Time-Dependent FMEA Model}

To propose a model of the hierarchical FMEA, we assume the following conditions: (i) All SSFs occur sequentially from bottom level to top level under the condition that the FC has already occurred, (ii) the occurrence of SSF follows the homogenous Poisson process, (iii) the detection time is followed by a uniform distribution before and exponential distribution after an SF, (iv) the repair costs for SSF are constant, and (vi) the opportunity cost due to the effect of system failure increases proportionally with system downtime.

Based on these assumptions, we first define the loss function to consider the situation in which the loss varies according to the detection time. Second, the probability density function of FC detection is defined under the assumption that the function will be different before and after the SF. Third, the probability density function of failure occurrence for the SSF is defined and then, based on the previously defined probability function, the expected value of the loss due to the FC is calculated.

In this paper, we set a system consisting of an $(n+2)$-layered hierarchy, where the top system is the 0th level and the FC occurs at level $n+1$.

\subsection{Separation of a Loss Function}

The loss can be considered as the point whether the FC is detected before the SF or not; (i) detected before an SF and (ii) detected after an SF. In general, if an FC is found and repaired before an SF occurs, there will be no additional loss due to the elapsed time from the time of the occurrence of the cause to the time of the detection of the cause, other than the cost of repairing the cause. Therefore, in case (i), the cost of repairing the SSF can be set to a constant value $\alpha$. On the other hand, if the FC is searched after the SF, as in case (ii), the opportunity cost will be incurred due to the production interruption, due to the system inoperability. Since this will be proportional to the system downtime, $s-t_{0}(s$ is the detection time and $t_{0}$ is the SF time), we set the variable cost to $\theta\left(s-t_{0}\right)$ according to the system downtime. In addition, the fixed cost will be added to repair the SSF and SF. If we set the repair cost of SF be $\beta$, the repair cost of SSF and SF would be $\alpha+\beta$.

Now, we separate the loss function in two functions such that $L_{1}\left(s, t_{0}\right)$ and $L_{2}\left(s, t_{0}\right)$. First, we set $L_{1}\left(s, t_{0}\right)$ be the repair cost of the SSF, which is trivially a constant $\alpha$ regardless of the detection time. Second, we set $L_{2}\left(s, t_{0}\right)$ to be the repair cost of the SF and the opportunity cost of the system downtime. If the detection time, $s$, is before the SF time, $t_{0}, L_{2}\left(s, t_{0}\right)$ is 0 . Otherwise, $L_{2}\left(s, t_{0}\right)$ is the sum of the repair cost of $\mathrm{SF}, \beta$, and the opportunity cost, $\theta\left(s-t_{0}\right)$, of system downtime. Thus, the loss function, $L\left(s, t_{0}\right)$, is expressed as follows:

$$
L\left(s, t_{0}\right)=L_{1}\left(s, t_{0}\right)+L_{2}\left(s, t_{0}\right)
$$

where

$\mathrm{s}$ is the detection time of the FC $\left(n+1^{\text {th }}\right.$ level); and

$t_{0}$ is the failure time of the system $\left(0^{\text {th }}\right.$ level),

with

$$
\begin{gathered}
L_{1}\left(s, t_{0}\right)=\alpha, \\
L_{2}\left(s, t_{0}\right)=\left\{\begin{array}{ll}
0, & s<t_{0} \\
\beta+\theta\left(s, t_{0}\right), & s \geq t_{0}
\end{array} .\right.
\end{gathered}
$$




\subsection{Detection of the Failure-Cause (FC)}

As the loss function is separated by detection before and after the SF, we need to consider the separation of the detection function before and after the SF. It is not easy to recognize the FC until the system stops operating due to the $0^{\text {th }}$ level failure. Therefore, the probability that the detection occurs before the SF time $t_{0}$ is uniform with the inspection schedule. However, if the $0^{\text {th }}$ level failure occurs and the system stops operating, this method will focus on finding the FC regardless of the detecting schedule. Therefore, the detection probability of the FC will jump up right after the SF and decrease after jumping. Assuming that detection is based on the memory-less property, we can easily estimate that the probability of detection after an SF is exponential.

Figure 3 expresses the probability density function of the FC detection in a case function. Let $w\left(t_{0}\right)$ be the probability of uniform distribution, with $s<t_{0}$, and let $1-w\left(t_{0}\right)$ be that of the exponential distribution. That is, $P\left(S<t_{0}\right)=w\left(t_{0}\right)$ and $P\left(S \geq t_{0}\right)=1-w\left(t_{0}\right)$. Then, the probability density function, $g_{S \mid T_{0}=t_{0}}(\mathrm{~s})$, becomes a case function such that

$$
g_{S \mid T_{0}=t_{0}}(s)=\left\{\begin{array}{ll}
w\left(t_{0}\right) \frac{1}{t_{0}}, & s<t_{0} \\
{\left[1-w\left(t_{0}\right)\right] \mu e^{-\mu\left(s-t_{0}\right)},} & s \geq t_{0}
\end{array} .\right.
$$

Suppose the detection probability is $c$ times jumping up at the time $s=t_{0}$ of the SF. Thus,

$$
c w\left(t_{0}\right) \frac{1}{t_{0}}=\left[1-w\left(t_{0}\right)\right] \mu e^{-\left(t_{0}-t_{0}\right)}=\left[1-w\left(t_{0}\right)\right] \mu .
$$

Hence,

$$
w\left(t_{0}\right)=\frac{\mu t_{0}}{c+\mu t_{0}}
$$

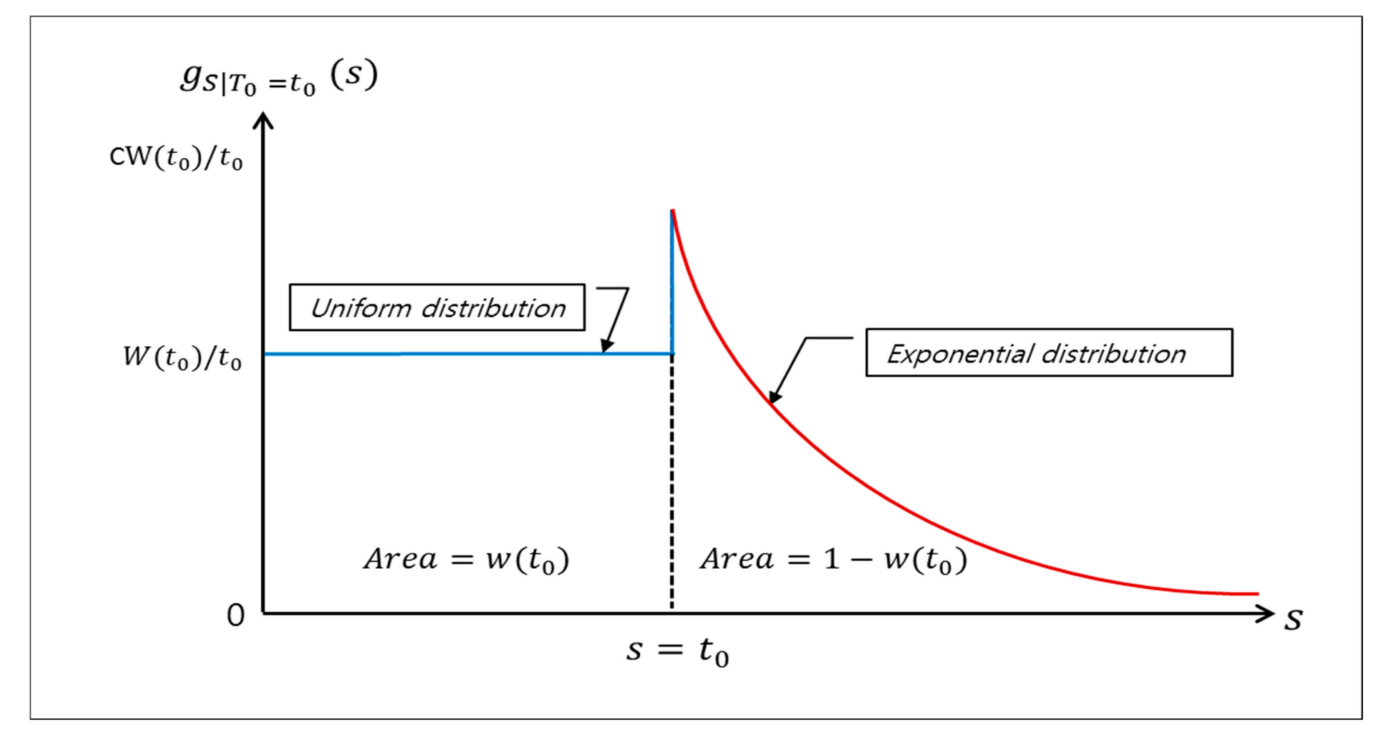

Figure 3. Probability density function of FC detection, $g_{S \mid T_{0}=t_{0}}(s)$, conditioned by the SF time $T_{0}=t_{0}$.

The weight function equals zero where $t_{0}$ is zero and becomes one as $t_{0}$ approaches infinity. This is very reasonable because the probability of detection before the failure is zero where $t_{0}$ is zero, and that of detection before the failure becomes one as $t_{0}$ approaches infinity. Therefore,

$$
g_{S \mid T_{0}=t_{0}}(s)=\left\{\begin{array}{ll}
\frac{\mu}{c+\mu t_{0}}, & s<t_{0} \\
\frac{c \mu}{c+\mu t_{0}} e^{-\mu\left(s-t_{0}\right)}, & s \geq t_{0}
\end{array} .\right.
$$




\subsection{Occurrence of a Subsystem Failure (SSF) of a Multi-Layered Structure}

In this paper, we deal with the $(n+2)$-layered system, and the loss function is analyzed from the point of the time that the bottom-level subsystem, i.e., the $(n+1)^{\text {th }}$ layer subsystem or FC, failure occurs. We assume that failures occur sequentially from the bottom level to the top level and follow the homogeneous Poisson process (HPP). Thus, the probability density function of the $n^{\text {th }}$ level SSF has a simple exponential function. Then, the probability density function of $(n+1)^{\text {th }}$ level SSF is a conditioned exponential function with the $n^{\text {th }}$ level failure time $t_{n}$. This process is continued to the $0^{\text {th }}$ level SF. Thus, each $k^{\text {th }}$ level probability density function, $h_{T_{k} \mid T_{k+1}=t_{k+1}}^{k}\left(t_{k}\right)$, is

$$
\begin{aligned}
& h_{T_{n}}^{n}\left(t_{n}\right)=\lambda_{n} e^{-\lambda_{n} t_{n}} ; t_{n} \geq 0 \\
& h_{T_{n-1} \mid T_{n}=t_{n}}^{n-1}\left(t_{n-1}\right)=\lambda_{n-1} e^{-\lambda_{n-1}\left(t_{n-1}-t_{n}\right)} ; t_{n-1} \geq t_{n} \\
& \ldots \\
& h_{T_{0} \mid T_{1}=t_{1}}^{0}\left(t_{0}\right)=\lambda_{0} e^{-\lambda_{0}(t 0-t 1)} ; t_{0} \geq t_{1} .
\end{aligned}
$$

Since we assume the $(n+1)^{\text {th }}$ subsystem (i.e., FC) has already failed at time $t_{n+1}=0$, we do not consider the probability density function of the $(n+1)^{\text {th }}$ SSF. Then, the probability density function of the system failure is

$$
\begin{aligned}
h_{T_{0}}\left(t_{0}\right) & =h_{T_{0} \mid T_{1}=t_{1}}^{0}\left(t_{0}\right) \cdot \ldots \cdot h_{T_{n-1} \mid T_{n}=t_{n}}^{n-1}\left(t_{n-1}\right) \cdot h_{T_{n}}^{n}\left(t_{n}\right) \\
& =\lambda_{0} e^{-\lambda_{0}\left(t_{0}-t_{1}\right)} \cdot \ldots \cdot \lambda_{n-1} e^{-\lambda_{n-1}\left(t_{n-1}-t_{n}\right)} \cdot \lambda_{n} e^{-\lambda_{n}\left(t_{n}\right)} .
\end{aligned}
$$

\subsection{Joint Probability Function of an Occurrence and Detection of Subsystem Failure (SSF)}

In Section 3.1, Equations (1)-(3) express a loss function as the sum of the repair cost of FC, the repair cost of SF, and the opportunity cost of the system downtime. As the loss function is a function of the detection time, $s$, and SF time, $t_{0}$, the joint probability density function of the occurrence and detection of SSF is the product of the detection function (7) and occurrence function (9).

$$
f_{S}, T_{0}, T_{1}, \cdots, T_{n}\left(s, t_{0}, t_{1}, \cdots, t_{n}\right)=g_{S \mid T_{0}=t_{0}}(s) h_{T_{0}}\left(t_{0}\right) .
$$

\subsection{Evaluation of Losses Due to Failure-Cause (FC)}

As introduced in Section 3.2, the loss function is separated by two functions, $L_{1}\left(s, t_{0}\right)$ and $L_{2}\left(s, t_{0}\right)$, so we can also evaluate the expected value of the loss separately. That is,

$$
E[L]=E\left[L_{1}\right]+E\left[L_{2}\right] .
$$

Let $\vec{t}=\left(t_{0}, \cdots, t_{n-1}, t_{n}\right)$ be a vector in the set $\Omega \subset \mathbb{R}^{n+1}$ satisfying $t_{0} \geq \cdots \geq t_{n-1} \geq t_{n} \geq$ $t_{n+1}=0$. Let $\vec{T}=\left(T_{0}, \cdots, T_{n-1}, T_{n}\right)$ be a vector of the random variables of the failure occurrence for each subsystem.

Then,

$$
\begin{aligned}
E\left[L_{1}\right] & =\int_{\Omega} \int_{0}^{\infty} L_{1}\left(s, t_{0}\right) f_{s, \vec{T}}(s, \vec{t}) d s d \vec{t} \\
& =\alpha \int_{\Omega} \int_{0}^{\infty} f_{S, \vec{T}}(s, \vec{t}) d s d \vec{t} \\
& =\alpha
\end{aligned}
$$


The integration of the probability density function in the whole range is

$$
\int_{\Omega} \int_{0}^{\infty} f_{S, \vec{T}}(s, \vec{t}) d s d \vec{t}=1
$$

Furthermore,

$$
\begin{aligned}
E\left[L_{2}\right]= & \int_{\Omega} \int_{0}^{\infty} L_{2}\left(s, t_{0}\right) f_{S, \vec{T}}(s, \vec{t}) d s d \vec{t} \\
& ; L_{2}\left(s, t_{0}\right)=0 \text { for } s<t_{0} \\
= & \int_{\Omega} \int_{t_{0}}^{\infty} L_{2}\left(s, t_{0}\right) f_{S, \vec{T}}(s, \vec{t}) d s d \vec{t} \\
= & \int_{\Omega} \int_{t_{0}}^{\infty} L_{2}\left(s, t_{0}\right) g_{S \mid T_{0}=t_{0}}(s) h_{T_{0}}\left(t_{0}\right) d s d \vec{t} \\
= & \int_{\Omega} \int_{t_{0}}^{\infty} L_{2}\left(s, t_{0}\right) g_{S \mid T_{0}=t_{0}}(s) h_{T_{0} \mid T_{1}=t_{1}}^{0}\left(t_{0}\right) \cdot \ldots \cdot h_{T_{n-1} \mid T_{n}=t_{n}}^{n-1}\left(t_{n-1}\right) \cdot h_{T_{n}}^{n}\left(t_{n}\right) d s d \vec{t} \\
= & \int_{0}^{\infty} \int_{t_{n}}^{\infty} \cdots \int_{t_{1}}^{\infty} \int_{t_{0}}^{\infty}\left[\beta+\theta\left(s-t_{0}\right)\right] \frac{c \mu}{c+\mu t_{0}} e^{-\mu\left(s-t_{0}\right)} \lambda_{0} e^{-\lambda_{0}\left(t_{0}-t_{1}\right)} \ldots \\
& \quad \cdot \lambda_{n-1} e^{-\lambda_{n-1}\left(t_{n-1}-t_{n}\right)} \cdot \lambda_{n} e^{-\lambda_{n} t_{n}} d s d t_{0} \ldots d t_{n-1} d t_{n} \\
= & \left(\beta+\frac{\theta}{\mu}\right) \cdot \frac{c}{\mu} \prod_{i=0}^{n} \lambda_{i} \cdot \sum_{j=0}^{n}\left[\frac{e^{\frac{c \lambda_{j}}{\mu}} E_{1}\left(\frac{c \lambda_{j}}{\mu}\right)}{\prod_{k \neq j}^{n}\left(\lambda_{k}-\lambda_{j}\right)}\right]
\end{aligned}
$$

(See Appendix A)

Here, $E_{1}(x)=\int_{x}^{\infty} \frac{e^{-t}}{t} d t=-\gamma-\ln x-\sum_{k=1}^{\infty} \frac{(-x)^{k}}{k \cdot k !} \quad$ is a special function named as exponential integral.

\section{Example Cases}

The functional level FTA depicted in Figure 1 was adopted as a case study to demonstrate the utility of the proposed time-dependent probabilistic model for a hierarchical structure. Based on

\begin{tabular}{|c|c|c|c|c|c|c|c|}
\hline & & tem Failure & $\beta$ & Failure Effect & $\theta$ & \multirow{2}{*}{\multicolumn{2}{|c|}{$E[L]$}} \\
\hline \multicolumn{3}{|c|}{ Powder Layer Deposit Failure } & 700 & Print Operation Failure & 1000 & & \\
\hline CASE & Level & Subsystem Failure & $\lambda$ & $\mu$ & $\alpha$ & $\mathrm{c}=1$ & $c=5$ \\
\hline A & $\begin{array}{l}\text { 1st } \\
\text { 2nd } \\
\text { 3rd }\end{array}$ & $\begin{array}{l}\text { No complete layer } \\
\text { Recoater does not move } \\
\text { Recoater encoder failure }\end{array}$ & $\begin{array}{l}5.850 \times 10^{-1} \\
3.611 \times 10^{-3} \\
7.215 \times 10^{-4}\end{array}$ & $2.686 \times 10^{-2}$ & 300 & 2030 & 6730 \\
\hline B & $\begin{array}{l}\text { 1st } \\
\text { 2nd } \\
\text { 3rd } \\
4 \text { th }\end{array}$ & $\begin{array}{l}\text { No complete layer } \\
\text { Piston does not move } \\
\text { Drive train failure } \\
\text { Pulley failure }\end{array}$ & $\begin{array}{l}7.400 \times 10^{-2} \\
3.611 \times 10^{-3} \\
1.926 \times 10^{-1} \\
5.850 \times 10^{-1}\end{array}$ & $7.648 \times 10^{-1}$ & 500 & 523 & 602 \\
\hline
\end{tabular}
Figures 1 and 2, the HTD-FMEA sheet was constructed as Table 1. Numerical values were randomly assigned to parameters as follows: (i) The constant cost, $\beta=700$, of repairing the SF; (ii) the opportunity cost, $\theta=1,000$, of the failure effect due to "print operation failure"; (iii) the occurrence rate $\lambda$ of each SSF; (iv) the detection rate $\mu$ of the FC; (v) the constant cost $\alpha$ of repairing the SSF; and (vi) the detection probability increment coefficient $\mathrm{c}$ at the time of the SF. It can be utilized as a maintenance policy coefficient. If $\mathrm{c}$ is set to more than 1 , the current maintenance policy is focused on post maintenance rather than preventive maintenance. Here, $c$ is set to 1 and 5 .

Table 1. HTD-FMEA sheet from the example in Figure 2. 
In Table 1, Case A shows that the $E[L]$ in a situation where the "Recoater encoder failure" is detected after the occurrence of the "Powder layer deposit failure" is calculated by applying Equation (A24). When $\mathrm{c}=1$, the $E[L]$ is 2030, which is a predictable loss that reflects the values of $\alpha, \beta$, and $\theta$ in proportion to the detection probability. On the other hand, when $c=5$, the $E[L]$ is 6730 , which shows that the expected value of the loss is more than three times that of $\mathrm{c}=1$. The reason for this difference is that the detecting system for the "Recoater encoder failure" is not only very weak but, additionally, the maintenance policy is focused on post-maintenance, resulting in an increase in the opportunity cost $\theta$.

Case B shows that the $E[L]$ in a situation where the "Pulley failure" was detected after the occurrence of the "Powder layer deposit failure" is calculated by applying Equation (A25). The $E[L]$ values were 523 and 602 for $\mathrm{c}=1$ and 5 , respectively. In this case, the detection system for the "Pulley failure" is well-equipped and, even if the maintenance policy is focused on post-maintenance, $E[L]$ is not expected to increase significantly.

In this case, the loss due to Case A is expected to be much larger, so it is necessary to improve the direction of reducing the occurrence of the "Recoater encoder failure" or strengthening the detection system. At this time, it is desirable to take remedial measures to further reduce the loss.

\section{Discussion}

This paper suggests a novel hierarchical risk-evaluation model-time-dependent probabilistic loss model — to mitigate the drawbacks of the RPN approach employed in conventional FMEA. The risk to the FCs can be estimated through $E[L]$ or the expected loss per unit time, which can be obtained by multiplying the FC's occurrence rate per unit time with the corresponding value of $E[L]$. The key benefits of our model are that (i) the $E[L]$ of any level in hierarchical failure can be calculated by using the proposed model; (ii) the $E[L]$ corresponds to an absolute loss-magnitude criterion that does not require other interpretations, i.e., the engineer can easily compare the risk of each failure based on the $E[L]$, whereas RPN values only provide comparative priority information that may require further assessment to be performed; and (iii) it is possible to predict how $E[L]$ will decrease in situations where the occurrence rate or detection power is improved when the corrective action is performed on a FC with a large $E[L]$. An engineer may also be able to establish a maintenance policy based on the loss in the expected savings.

This study is expected to be applicable to the risk evaluation of hierarchical manufacturing systems in the field of advanced production technology. To demonstrate the practicality of this study in the future, case studies will be conducted based on actual failure data and maintenance costs. Based on this study, an extension study will be carried out on the established loss minimization maintenance policy in FMEA.

Author Contributions: Conceptualization, H.J. and S.M.; methodology, H.J. and S.M.; modeling, S.M.; validation, H.J. and S.M.; writing_-original draft preparation, H.J. and S.M.; writing—review and editing, H.J. and SM.; funding acquisition, H.J.

Funding: This work was supported by the National Research Foundation of Korea (NRF) in a grant funded by the Korean government (MSIT) (No. 2019R1G1A1010335 and 2017R1C1B5017491).

Conflicts of Interest: The authors declare no conflicts of interest.

\section{Appendix A}

To evaluate the loss of a hierarchical structure in our model, we use some formulae about the exponential integral, as follows [40]. 
Table A1. Useful formulae about exponential integral to evaluate the loss.

\begin{tabular}{cc}
\hline Name & Equation \\
\hline $\begin{array}{c}\text { Exponential integral } \\
E_{1}(x)=\int_{x}^{\infty} \frac{e^{-t}}{t} d t=-\gamma-\ln x-\sum_{k=1}^{\infty} \frac{(-x)^{k}}{k \cdot k !}\end{array}$ \\
$\begin{array}{c}\text { where } \gamma=\lim _{n \rightarrow \infty}\left(\sum_{k=1}^{n} \frac{1}{k}-\ln n\right)=0.5772156649 \ldots . \text { is } \\
\text { the Euler constant. }\end{array}$ \\
\hline $\begin{array}{c}\text { Integration of exponential integral combined by } \\
\text { exponential function }\end{array}$ & $\begin{array}{c}\int e^{-a x} E_{1}(b x) d x=\frac{1}{a}\left\{E_{1}[(a+b) x]-e^{-a x} E_{1}(b x)\right\}, \text { so } \\
\int_{x}^{\infty} e^{-a \chi} E_{1}(b x) d \chi=\frac{1}{a}\left\{e^{-a x} E_{1}(b x)-E_{1}[(a+b) x]\right\} .\end{array}$ \\
\hline
\end{tabular}

In addition, we define $l_{k}$ and $\tau_{k}$ as follows for the sake of convenience.

$$
\begin{gathered}
l_{k}:=\lambda_{k} / \lambda_{0} \quad ; l_{0}=1, \\
\tau_{k}:=c \lambda_{0} / \mu+\lambda_{0} t_{k}=\lambda_{0}\left(c / \mu+t_{k}\right),
\end{gathered}
$$

where $k$ is any non-negative integer such that $0 \leq k \leq n+1$

Especially, $\tau_{n+1}=c \lambda_{0} / \mu$, since we consider that the time from the $n+1$ subsystem is failed, i.e., $t_{k+1}=0$. To reduce the confusion, we define $\tau_{\infty}\left(=\tau_{n+1}\right.$ for $(n+2)$-layered structure $)$ as the following.

$$
\tau_{\infty}:=c \lambda_{0} / \mu
$$

Then, for the given $t_{k+1}\left(\leq t_{k}\right)$, the following equations are easily derived.

$$
\lambda_{k}\left(t_{k}-t_{k+1}\right)=l_{k}\left(\tau_{k}-\tau_{k+1}\right)
$$

Now, for $t_{k} \geq t_{k+1}$, the conditioned probability density function of the $k^{\text {th }}$ subsystem failure can be changed as the simplified form

$$
h_{T_{k} \mid T_{k+1}=t_{k+1}}^{k}\left(t_{k}\right) d t_{k}=\lambda_{K} e^{-\lambda_{k}\left(t_{k}-t_{k+1}\right)} d t_{k}=l_{k} e^{-l_{k}\left(\tau_{k}-\tau_{k+1}\right)} d \tau_{k}
$$

Lemma 1. Let $\vec{t}=\left(t_{0}, \ldots, t_{n-1}, t_{n}\right) \in \Omega\left(\subset \mathbb{R}^{n+1}\right)$ be a vector satisfying that $t_{0} \geq \ldots \geq t_{n-1} \geq t_{n} \geq 0$. Then the expected value $E\left[L_{2}\right]$ is as follows:

$$
E\left[L_{2}\right]=(\beta+\theta / \mu) \int_{\Omega} \frac{c}{c+\mu t_{0}} h_{T_{0}}\left(t_{0}\right) d \vec{t} .
$$




\section{Proof}

$$
\begin{aligned}
E\left[L_{2}\right] & =\int_{\Omega} \int_{0}^{\infty} L_{2}\left(s, t_{0}\right) f_{S, \vec{T}}(s, \vec{t}) d s d \vec{t} \\
& =\int_{\Omega} \int_{t_{0}}^{\infty} L_{2}\left(s, t_{0}\right) g_{S \mid T_{0}=t_{0}}(s) h_{T_{0}}\left(t_{0}\right) d s d \vec{t} \\
& =\int_{\Omega} \int_{t_{0}}^{\infty}\left[\beta+\theta\left(s-t_{0}\right)\right]\left[\frac{c \mu}{c+\mu t_{0}} e^{-\mu\left(s-t_{0}\right)}\right] h_{T_{0}}\left(t_{0}\right) d s d \vec{t} \\
& =\int_{\Omega}\left\{\int_{t_{0}}^{\infty}\left[\beta+\theta\left(s-t_{0}\right)\right] \mu e^{-\mu\left(s-t_{0}\right)} d s\right\} \frac{c}{c+\mu t_{0}} h_{T_{0}}\left(t_{0}\right) d \vec{t}\left\{\int_{t_{0}}^{\infty}\left[\beta+\theta\left(s-t_{0}\right)\right] \mu e^{-\mu\left(s-t_{0}\right)} d s\right\} \\
& =\int_{0}^{\infty}(\beta+\theta \sigma) \mu e^{-\mu \sigma} d \sigma \\
& \quad ; \sigma=s-t_{0} \\
& =\left[\left.(\beta+\theta \sigma)\left(-e^{-\mu \sigma}\right)\right|_{0} ^{\infty}-\left.\theta\left(-e^{-\mu \sigma} / \mu\right)\right|_{0} ^{\infty}\right] \\
& \quad ; \text { integration by parts } \\
& =(\beta+\theta / \mu \quad \theta / \mu) \int_{\Omega} \frac{c}{c+\mu t_{0}} h_{T_{0}}\left(t_{0}\right) d \vec{t} .
\end{aligned}
$$

Lemma 2. Suppose that $l_{k} \neq l_{j}$ where $k \neq j$. Then for any positive integer $n \geq 1$,

$$
\sum_{j=0}^{n-1}\left[\frac{1}{\prod_{k \neq j}^{n-1}\left(l_{k}-l_{j}\right)\left(l_{j}-l_{n}\right)}\right]=\left[\frac{1}{\prod_{k=0}^{n-1}\left(l_{k}-l_{n}\right)}\right] .
$$

Here, the product of the empty number is defined 1 as a summation of that is 0 , i.e.,

$$
\begin{gathered}
\prod_{c_{i} \in \varnothing} c_{i}=1 \text { likewise } \\
\sum_{c_{i} \in \varnothing} c_{i}=0
\end{gathered}
$$

Proof. In a special case, for $n=1$

$$
\sum_{j=0}^{n-1}\left[\frac{1}{\prod_{k \neq j}^{n-1}\left(l_{k}-l_{j}\right)\left(l_{j}-l_{n}\right)}\right]=\frac{1}{l_{0}-l_{n}}=\left[\frac{1}{\prod_{k=0}^{n-1}\left(l_{k}-l_{n}\right)}\right] .
$$

Now, for $n \geq 2$, define $f(x)$ and $g(x)$ be a function of an independent variable $x$, such that

$$
f(x)=\sum_{j=0}^{n-1}\left[\frac{1}{\prod_{k \neq j}^{n-1}\left(l_{k}-l_{j}\right)\left(l_{j}-x\right)}\right],
$$

and

$$
g(x)=f(x) \prod_{k=0}^{n-1}\left(l_{k}-x\right)-1
$$


Then $g(x)$ is an $n-1^{\text {th }}$ order polynomial such that

$$
\begin{aligned}
& g(x)=\sum_{j=0}^{n-1}\left[\prod_{k \neq j}^{n-1} \frac{\left(l_{k}-x\right)}{\left(l_{k}-l_{j}\right)}\right]-1 \\
& =\frac{\left(l_{1}-x\right) \ldots\left(l_{n-1}-x\right)}{\left(l_{1}-l_{0}\right) \ldots\left(l_{n-1}-l_{0}\right)}+\ldots+\frac{\left(l_{0}-x\right) \ldots\left(l_{n-2}-x\right)}{\left(l_{0}-l_{n-1}\right) \ldots\left(l_{n-2}-l_{n-1}\right)}-1 .
\end{aligned}
$$

We can easily deduce the following by substituting $x=l_{0}, \cdots, x=l_{n-1}$

$$
g\left(l_{0}\right)=\ldots=g\left(l_{n-1}\right)=0
$$

The $n-1^{\text {th }}$ order polynomial has at most $n-1$ zeros, but $g(x)$ has $n$ zeros such that $x=l_{0}, \cdots, x=l_{n-1}$. Thus, we can conclude $g(x)$ is constantly zero.

Hence,

$$
\begin{gathered}
g\left(l_{n}\right)+1=f\left(l_{n}\right) \prod_{k=0}^{n-1}\left(l_{k}-l_{n}\right)=1, \text { i.e., } \\
f\left(l_{n}\right)=\left[\frac{1}{\prod_{k=0}^{n-1}\left(l_{k}-l_{n}\right)}\right]
\end{gathered}
$$

Lemma 3. For any positive number $n \geq 1$,

$$
\int_{t_{n}}^{\infty} \cdots \int_{t_{1}}^{\infty} \frac{c}{c+\mu t_{0}} \lambda_{0} e^{-\lambda_{0}\left(t_{0}-t_{1}\right)} \cdot \ldots \cdot \lambda_{n-1} e^{-\lambda_{n-1}\left(t_{n-1}-t_{n}\right)} d t_{0} \ldots d t_{n-1}=\tau_{\infty} \prod_{i=1}^{n-1} l_{i} \cdot \sum_{j=0}^{n-1}\left[\frac{e^{l_{j} \tau_{n}} E_{1}\left(l_{j} \tau_{n}\right)}{\prod_{k \neq j}^{n-1}\left(l_{k}-l_{j}\right)}\right]
$$

Proof. Define $a_{n}$ be $\int_{t_{n}}^{\infty} \cdots \int_{t_{1}}^{\infty} \frac{c}{c+\mu t_{0}} \lambda_{0} e^{-\lambda_{0}\left(t_{0}-t_{1}\right)} \cdot \ldots \cdot \lambda_{n-1} e^{-\lambda_{n-1}\left(t_{n-1}-t_{n}\right)} d t_{0} \ldots d t_{n-1}$ for $n \geq 1$. Then

$$
\begin{gathered}
a_{n+1}=\int_{t_{n+1}}^{\infty} a_{n} \lambda_{n} e^{-\lambda_{n}\left(t_{n}-t_{n+1}\right)} d t_{n} \\
=\int_{\tau_{n+1}}^{\infty} a_{n} l_{n} e^{-l_{n}\left(\tau_{n}-\tau_{n+1}\right)} d \tau_{n} .
\end{gathered}
$$

We can carry out the proof by mathematical induction. For $N=1$,

$$
\begin{aligned}
& a_{1}=\int_{t_{1}}^{\infty} \frac{c}{c+\mu t_{0}} \lambda_{0} e^{-\lambda_{0}\left(t_{0}-t_{1}\right)} d t_{0} \\
& \quad=\int_{\tau_{1}}^{\infty} \frac{\tau_{\infty}}{\tau_{0}} e^{-\left(\tau_{0}-\tau_{1}\right)} d \tau_{0} \\
& \quad ; \tau_{0}=c \lambda_{0} / \mu+\lambda_{0} t_{0} \text { and } d \tau_{0}=\lambda_{0} d t_{0} \\
& \quad=\tau_{\infty} e^{\tau_{1}}=\int_{\tau_{1}}^{\infty} \frac{e^{-\tau_{0}}}{\tau_{0}} d \tau_{0} \\
& =\tau_{\infty} e^{l_{0} \tau_{1}} E_{1}\left(l_{0} \tau_{1}\right) .
\end{aligned}
$$

$l_{0}=1$ and

$E_{1}(x)$ is the exponential integral as defined in the Table A1. 
Thus, Lemma 3 satisfies for $n=1$. Now, suppose the proposition suffices for $n \in \mathbb{N}$, then for $n+1$,

$$
\begin{aligned}
& a_{n+1}=\int_{\tau_{n+1}}^{\infty} a_{n} l_{n} e^{-l_{n}\left(\tau_{n}-\tau_{n+1}\right)} d \tau_{n} \\
& =\int_{\tau_{n+1}}^{\infty}\left\{\tau_{\infty} \prod_{i=1}^{n-1} l_{i} \cdot \sum_{j=0}^{n-1}\left[\frac{e^{l_{j} \tau_{n}} E_{1}\left(l_{j} \tau_{n}\right)}{\prod_{k \neq j}^{n-1}\left(l_{k}-l_{j}\right)}\right] \cdot l_{n} e^{-l_{n}\left(\tau_{n}-\tau_{n+1}\right)}\right\} d \tau_{n} \\
& =\tau_{\infty} e^{l_{n} \tau_{n+1}} \prod_{i=1}^{n} l_{i} \cdot \sum_{j=0}^{n-1}\left[\frac{\int_{\tau_{n+1}}^{\infty} e^{-\left(l_{n}-l_{j}\right) \tau_{n}} E_{1}\left(l_{j} \tau_{n}\right) d \tau_{n}}{\prod_{k \neq j}^{n-1}\left(l_{k}-l_{j}\right)}\right] \\
& =\tau_{\infty} e^{l_{n} \tau_{n+1}} \prod_{i=1}^{n} l_{i} \cdot \sum_{j=0}^{n-1}\left[\frac{\frac{1}{l_{n}-l_{j}}\left\{e^{-\left(l_{n}-l_{j}\right) \tau_{n+1}} E_{1}\left(l_{j} \tau_{n+1}\right)-E_{1}\left(l_{n} \tau_{n+1}\right)\right\}}{\prod_{k \neq j}^{n-1}\left(l_{k}-l_{j}\right)}\right] ; \\
& \int_{x}^{\infty} e^{-a \chi} E_{1}(b x) d \chi=\frac{1}{a}\left\{e^{-a x} E_{1}(b x)-E_{1}[(a+b) x]\right\} \text { with } \\
& a=l_{n}-l_{j}, b=l_{j} \text { and } x=\tau_{n+1} \\
& =\tau_{\infty} \prod_{i=1}^{n} l_{i} \cdot \sum_{j=0}^{n-1}\left[\frac{e^{l_{j} \tau_{n+1}} E_{1}\left(l_{j} \tau_{n+1}\right)}{\prod_{k \neq j}^{n-1}\left(l_{k}-l_{j}\right)\left(l_{n}-l_{j}\right)}\right]-\tau_{\infty} \prod_{i=1}^{n} l_{i} \cdot \sum_{j=0}^{n-1}\left[\frac{e^{l_{n} \tau_{n+1}} E_{1}\left(l_{n} \tau_{n+1}\right)}{\prod_{k \neq j}^{n-1}\left(l_{k}-l_{j}\right)\left(l_{n}-l_{j}\right)}\right] \\
& =\tau_{\infty} \prod_{i=1}^{n} l_{i} \cdot \sum_{j=0}^{n-1}\left[\frac{e^{l_{j} \tau_{n+1}} E_{1}\left(l_{j} \tau_{n+1}\right)}{\prod_{k \neq j}^{n}\left(l_{k}-l_{j}\right)}\right]+\tau_{\infty} \prod_{i=1}^{n} l_{i} \cdot \sum_{j=0}^{n-1}\left[\frac{e^{l_{n} \tau_{n+1}} E_{1}\left(l_{n} \tau_{n+1}\right)}{\prod_{k \neq j}^{n-1}\left(l_{k}-l_{j}\right)\left(l_{j}-l_{n}\right)}\right] \\
& =\tau_{\infty} \prod_{i=1}^{n} l_{i} \cdot \sum_{j=0}^{n-1}\left[\frac{e^{l_{j} \tau_{n+1}} E_{1}\left(l_{j} \tau_{n+1}\right)}{\prod_{k \neq j}^{n}\left(l_{k}-l_{j}\right)}\right]+\tau_{\infty} \prod_{i=1}^{n} l_{i} \cdot\left[\frac{e^{l_{n} \tau_{n+1} E_{1}\left(l_{n} \tau_{n+1}\right)}}{\prod_{k=0}^{n-1}\left(l_{k}-l_{n}\right)}\right] \\
& \text {; by Lemma } 2 \\
& =\tau_{\infty} \prod_{i=1}^{n} l_{i} \cdot \sum_{j=0}^{n-1}\left[\frac{e^{l_{j} \tau_{n+1}} E_{1}\left(l_{j} \tau_{n+1}\right)}{\prod_{k \neq j}^{n}\left(l_{k}-l_{j}\right)}\right]+\tau_{\infty} \prod_{i=1}^{n} l_{i} \cdot \sum_{j=n}^{n}\left[\frac{e^{l_{j} \tau_{j+1}} E_{1}\left(l_{j} \tau_{j+1}\right)}{\prod_{k \neq j}^{n}\left(l_{k}-l_{j}\right)}\right] \\
& =\tau_{\infty} \prod_{i=1}^{n} l_{i} \cdot \sum_{j=0}^{n}\left[\frac{e^{l_{j} \tau_{n+1}} E_{1}\left(l_{j} \tau_{n+1}\right)}{\prod_{k \neq j}^{n}\left(l_{k}-l_{j}\right)}\right] .
\end{aligned}
$$

Since the Lemma 3 suffices for $n+1$ if it does for $n \in \mathbb{N}$, the mathematical induction is cleared.

Theorem 1. The expected value of $L_{2}$ for the $\mathrm{n}+$ 2-layered system $E\left[L_{2}\right]$ with $n \geq 0$ is as follows.

$$
E\left[L_{2}\right]=\left(\beta+\frac{\theta}{\mu}\right) \cdot \frac{c}{\mu} \prod_{i=0}^{n} \lambda_{i} \cdot \sum_{j=0}^{n}\left[\frac{e^{\frac{c \lambda_{j}}{\mu}} E_{1}\left(\frac{c \lambda_{j}}{\mu}\right)}{\prod_{k \neq j}^{n}\left(\lambda_{k}-\lambda_{j}\right)}\right] .
$$


Proof.

$$
\begin{aligned}
& E\left[L_{2}\right]=\int_{0}^{\infty} \int_{t_{n}}^{\infty} \cdots \int_{t_{1}}^{\infty} \int_{t_{0}}^{\infty}\left[\beta+\theta\left(s-t_{0}\right)\right] \frac{c \mu}{c+\mu t_{0}} e^{-\mu\left(s-t_{0}\right)} \lambda_{0} e^{-\lambda_{0}\left(t_{0}-t_{1}\right)} \ldots \\
& \cdot \lambda_{n-1} e^{-\lambda_{n-1}\left(t_{n-1}-t_{n}\right)} \cdot \lambda_{n} e^{-\lambda_{n} t_{n}} d s d t_{0} \ldots d t_{n-1} d t_{n} \\
& =(\beta+\theta / \mu) \int_{t_{n+1}}^{\infty} \cdots \int_{t_{1}}^{\infty} \frac{c}{c+\mu t_{0}} \lambda_{0} e^{-\lambda_{0}\left(t_{0}-t_{1}\right)} \cdot \ldots \cdot \lambda_{n} e^{-\lambda_{n}\left(t_{n}-t_{n+1}\right)} d t_{0} \ldots d t_{n} \\
& \text {; by lemma } 1 \text { with } t_{n+1}=0 \\
& =(\beta+\theta / \mu) \tau_{\infty} \prod_{i=1}^{n} l_{i} \cdot \sum_{j=0}^{n}\left[\frac{e^{l_{j} \tau_{\infty}} E_{1}\left(l_{j} \tau_{\infty}\right)}{\prod_{k \neq j}^{n}\left(l_{k}-l_{j}\right)}\right] \\
& \text {; by lemma } 3 \text { with } \tau_{n+1}=\tau_{\infty}=c \lambda_{0} / \mu \\
& =(\beta+\theta / \mu) \tau_{\infty} \prod_{i=1}^{n} l_{i} \cdot \sum_{j=0}^{n}\left[\frac{e^{l^{j} \tau_{\infty}} E_{1}\left(l_{j} \tau_{\infty}\right)}{\prod_{k \neq j}^{n}\left(l_{k}-l_{j}\right)}\right] \\
& =\left(\beta+\frac{\theta}{\mu}\right) \cdot \frac{c}{\mu} \prod_{i=0}^{n} \lambda_{i} \cdot \sum_{j=0}^{n}\left[\frac{e^{\frac{c \lambda_{j}}{\mu}} E_{1}\left(\frac{c \lambda_{j}}{\mu}\right)}{\prod_{k \neq j}^{n}\left(\lambda_{k}-\lambda_{j}\right)}\right] . \\
& ; l_{j} \tau_{\infty}=c \lambda_{j} / \mu
\end{aligned}
$$

Example 1. 1-layered structure (exceptional case)

In a 1-layered system, the cause is a system itself. Therefore, detection of the cause failure is only after the system failure, i.e., $s \geq t_{0}=0$. That is

$$
g_{S}(\mathrm{~s})=\mu e^{-\mu s} ; s \geq 0
$$

Hence

$$
\begin{aligned}
E\left[L_{2}\right] & =\int_{0}^{\infty} L_{2}\left(s, t_{0}\right) g_{S}(s) d s \\
& =\int_{0}^{\infty}\left(\beta+\theta_{S}\right) \mu e^{-\mu s} d s \\
& =\left.\left(\beta+\theta_{S}\right)\left(-e^{-\mu s}\right)\right|_{0} ^{\infty}-\left.\theta\left(-e^{-\mu s} / \mu\right)\right|_{0} ^{\infty} \\
& \quad ; \text { integration by parts } \\
=\beta+\frac{\theta}{\mu} &
\end{aligned}
$$

Example 2. 2-layered structure

$$
\begin{aligned}
& E\left[L_{2}\right]=\left(\beta+\frac{\theta}{\mu}\right) a_{1} \\
& =\left(\beta+\frac{\theta}{\mu}\right) \frac{c \lambda_{0}}{\mu} e^{\frac{c \lambda_{0}}{\mu}} E_{1}\left(\frac{c \lambda_{0}}{\mu}\right) .
\end{aligned}
$$

Example 3. 3-layered structure

$$
\begin{aligned}
& E\left[L_{2}\right]=\left(\beta+\frac{\theta}{\mu}\right) a_{2} \\
& =\left(\beta+\frac{\theta}{\mu}\right) \frac{c \lambda_{0} \lambda_{1}}{\mu}\left[\frac{e^{\frac{c \lambda_{0}}{\mu}} E_{1}\left(\frac{c \lambda_{0}}{\mu}\right)}{\lambda_{1}-\lambda_{0}}+\frac{e^{\frac{c \lambda_{1}}{\mu}} E_{1}\left(\frac{c \lambda_{1}}{\mu}\right)}{\lambda_{0}-\lambda_{1}}\right] .
\end{aligned}
$$


Example 4. 4-layered structure

$$
\begin{aligned}
& E\left[L_{2}\right]=\left(\beta+\frac{\theta}{\mu}\right) a_{3} \\
& =\left(\beta+\frac{\theta}{\mu}\right) \frac{c \lambda_{0} \lambda_{1} \lambda_{2}}{\mu}\left[\frac{e^{\frac{c \lambda_{0}}{\mu}} E_{1}\left(\frac{c \lambda_{0}}{\mu}\right)}{\left(\lambda_{1}-\lambda_{0}\right)\left(\lambda_{2}-\lambda_{0}\right)}+\frac{e^{\frac{c \lambda_{1}}{\mu}} E_{1}\left(\frac{c \lambda_{1}}{\mu}\right)}{\left(\lambda_{0}-\lambda_{1}\right)\left(\lambda_{2}-\lambda_{1}\right)}+\frac{e^{\frac{c \lambda_{2}}{\mu}} E_{1}\left(\frac{c \lambda_{2}}{\mu}\right)}{\left(\lambda_{0}-\lambda_{2}\right)\left(\lambda_{1}-\lambda_{2}\right)}\right] .
\end{aligned}
$$

Example 5. 5-layered structure

$$
\begin{aligned}
& E\left[L_{2}\right]=\left(\beta+\frac{\theta}{\mu}\right) a_{4} \\
&=\left(\beta+\frac{\theta}{\mu}\right) \frac{c \lambda_{0} \lambda_{1} \lambda_{2} \lambda_{3}}{\mu}\left[\frac{e^{\frac{c \lambda_{0}}{\mu}} E_{1}\left(\frac{c \lambda_{0}}{\mu}\right)}{\left(\lambda_{1}-\lambda_{0}\right)\left(\lambda_{2}-\lambda_{0}\right)\left(\lambda_{3}-\lambda_{0}\right)}+\frac{e^{\frac{c \lambda_{1}}{\mu}} E_{1}\left(\frac{c \lambda_{1}}{\mu}\right)}{\left(\lambda_{0}-\lambda_{1}\right)\left(\lambda_{2}-\lambda_{1}\right)\left(\lambda_{3}-\lambda_{1}\right)}\right. \\
&\left.\quad+\frac{e^{\frac{c \lambda_{2}}{\mu}} E_{1}\left(\frac{c \lambda_{2}}{\mu}\right)}{\left(\lambda_{0}-\lambda_{2}\right)\left(\lambda_{1}-\lambda_{2}\right)\left(\lambda_{3}-\lambda_{2}\right)}+\frac{e^{\frac{c \lambda_{3}}{\mu}} E_{1}\left(\frac{c \lambda_{3}}{\mu}\right)}{\left(\lambda_{0}-\lambda_{3}\right)\left(\lambda_{1}-\lambda_{3}\right)\left(\lambda_{2}-\lambda_{3}\right)}\right]
\end{aligned}
$$

\section{References}

1. Kim, K.O.; Zuo, M.J. General model for the risk priority number in failure mode and effects analysis. Reliab. Eng. Syst. Saf. 2018, 169, 321-329. [CrossRef]

2. Geum, Y.; Cho, Y.; Park, Y. A systematic approach for diagnosing service failure: Service-specific FMEA and grey relational analysis approach. Math. Comput. Model. 2011, 54, 3126-3142. [CrossRef]

3. Santhosh, D.; Vinodh, S. Application of FMEA to an automotive leaf spring manufacturing organization. TQM J. 2012, 24, 260-274.

4. Yazdi, M.; Daneshvar, S.; Setareh, H. An extension to Fuzzy Developed Failure Mode and Effects Analysis (FDFMEA) application for aircraft landing system. Saf. Sci. 2017, 98, 113-123. [CrossRef]

5. Wang, W.; Liu, X.; Qin, Y.; Fu, Y. A risk evaluation and prioritization method for FMEA with prospect theory and Choquet integral. Saf. Sci. 2018, 110, 152-163. [CrossRef]

6. Liu, H.; Liu, L.; Liu, N. Risk evaluation approaches in failure mode and effects analysis: A literature review. Expert Syst. Appl. 2013, 40, 828-838. [CrossRef]

7. Fattahi, R.; Khalilzadeh, M. Risk evaluation using a novel hybrid method based on FMEA, extended MULTIMOORA, and AHP methods under fuzzy environment. Saf. Sci. 2018, 102, 290-300. [CrossRef]

8. Mentes, A.; Ozen, E. A hybrid risk analysis method for a yacht fuel system safety. Saf. Sci. 2015, 79, 94-104. [CrossRef]

9. Zhou, Q.; Thai, V.V. Fuzzy and grey theories in failure mode and effect analysis for tanker equipment failure prediction. Saf. Sci. 2016, 83, 74-79. [CrossRef]

10. Kumar, A.M.; Rajakarunakaran, S.; Pitchipoo, P.; Vimalesan, R. Fuzzy based risk prioritisation in an auto LPG dispensing station. Saf. Sci. 2018, 101, 231-247. [CrossRef]

11. Rhee, S.J.; Ishii, K. Using cost based FMEA to enhance reliability and serviceability. Adv. Eng. Inform. 2003, 17, 179-188. [CrossRef]

12. Dong, C. Failure mode and effects analysis based on fuzzy utility cost estimation. Int. J. Qual. Reliab. Manag. 2007, 24, 958-971. [CrossRef]

13. Von Ahsen, A. Cost-oriented failure mode and effects analysis. Int. J. Qual. Reliab. Manag. 2008, 25, 466-476. [CrossRef]

14. Sant'Anna, A.P. Probabilistic priority numbers for failure modes and effects analysis. Int. J. Qual. Reliab. Manag. 2012, 29, 349-362. 
15. Certa, A.; Hopps, F.; Inghilleri, R.; la Fata, C.M. A Dempster-Shafer Theory-based approach to the Failure Mode, Effects and Criticality Analysis (FMECA) under epistemic uncertainty: Application to the propulsion system of a fishing vessel. Reliab. Eng. Syst. Saf. 2017, 159, 69-79. [CrossRef]

16. Huang, J.; Li, Z.; Liu, H. New approach for failure mode and effect analysis using linguistic distribution assessments and TODIM method. Reliab. Eng. Syst. Saf. 2017, 167, 302-309. [CrossRef]

17. Catelani, M.; Ciani, L.; Venzi, M. Failure modes, mechanisms and effect analysis on temperature redundant sensor stage. Reliab. Eng. Syst. Saf. 2018, 180, 425-433. [CrossRef]

18. Ding, X.; Su, Q.; You, J.; Liu, H. Improving risk evaluation in FMEA with a hybrid multiple criteria decision making method. Int. J. Qual. Reliab. Manag. 2015, 32, 763-782.

19. Mohsen, O.; Fereshteh, N. An extended VIKOR method based on entropy measure for the failure modes risk assessment-A case study of the geothermal power plant (GPP). Saf. Sci. 2017, 92, 160-172. [CrossRef]

20. Lo, H.; Liou, J.J.H.; Huang, C.; Chuang, Y. A novel failure mode and effect analysis model for machine tool risk analysis. Reliab. Eng. Syst. Saf. 2019, 183, 173-183. [CrossRef]

21. Yousefi, S.; Alizadeh, A.; Hayati, J.; Baghery, M. HSE risk prioritization using robust DEA-FMEA approach with undesirable outputs: A study of automotive parts industry in Iran. Saf. Sci. 2018, 102, 144-158. [CrossRef]

22. Kwon, H.M.; Hong, S.H.; Lee, M.K. An expected loss model for FMEA under periodic monitoring of failure causes. J. Korean Inst. Ind. Eng. 2013, 39, 143-148.

23. Jang, H.A.; Lee, M.K.; Hong, S.H.; Kwon, H.M. Risk Evaluation Based on the Hierarchical Time Delay Model in FMEA. J. Korean Soc. Qual. Manag. 2016, 44, 373-388. [CrossRef]

24. Guimarães, A.C.F.; Lapa, C.M.F.; Moreira, M. Fuzzy methodology applied to Probabilistic Safety Assessment for digital system in nuclear power plants. Nucl. Eng. Des. 2011, 241, 3967-3976. [CrossRef]

25. Sharma, R.K.; Sharma, P. System failure behavior and maintenance decision making using, RCA, FMEA and FM. J. Qual. Maint. Eng. 2010, 16, 64-88. [CrossRef]

26. Heeralal, G.; Sanjay, K.C. Criticality Assessment Models for Failure Mode Effects and Criticality Analysis Using Fuzzy Logic. IEEE Trans. Reliab. 2011, 60, 102-110.

27. Zaili, Y.; Steve, B.; Jin, W. Fuzzy rule-based Bayesian reasoning approach for prioritization of failures in FMEA. IEEE Trans. Reliab. 2008, 57, 517-528. [CrossRef]

28. Pillay, A.; Wang, J. Modified failure mode and effects analysis using approximate reasoning. Reliab. Eng. Syst. Saf. 2003, 79, 69-85. [CrossRef]

29. Jih, K.C. Utility Priority Number Evaluation for FMEA. J. Fail. Anal. Prev. 2007, 7, 321-328.

30. Allen, H.H.; Chia, W.H.; Tsai, C.K.; Wei, C.W. Risk evaluation of green components to hazardous substance using FMEA and FAHP. Expert Syst. Appl. 2009, 36, 7142-7147.

31. Zammori, F.; Gabbrielli, R. ANP/RPN: A Multi Criteria Evaluation of the Risk Priority Number. Qual. Reliab. Eng. Int. 2012, 28, 85-104. [CrossRef]

32. Peeters, J.F.W.; Basten, R.J.I.; Tinga, T. Improving failure analysis efficiency by combining FTA and FMEA in a recursive manner. Reliab. Eng. Syst. Saf. 2018, 172, 36-44. [CrossRef]

33. Braglia, M. MAFMA: Multi-attribute failure mode analysis. Int. J. Qual. Reliab. Manag. 2000, 17, 1017-1033. [CrossRef]

34. Carmignani, G. An integrated structural framework to cost-based FMECA: The priority-cost FMECA. Reliab. Eng. Syst. Saf. 2009, 94, 861-871. [CrossRef]

35. Jang, H.A.; Yun, W.Y.; Kwon, H.M. Risk Evaluation in FMEA when the Failure Severity Depends on the Detection Time. J. Korean Soc. Saf. 2016, 31, 136-142. [CrossRef]

36. Wada, H. Safety analysis methods and applications at the design stage of new product development-Introducing the FMFEA and S-H Matrix Method. ESPEC Technol. Rep. 2000, 10, 1-7.

37. Jang, H.A.; Hong, S.H.; Lee, M.K.; Kwon, H.M. Risk evaluation of failure modes due to hierarchic failure causes in fmea. ICIC Express Lett. Part B Appl. Int. J. Res. Surv. 2015, 6, 383-390.

38. Carpitella, S.; Certa, A.; Izquierdo, J.; la Fata, C.M. A combined multi-criteria approach to support FMECA analyses: A real-world case. Reliab. Eng. Syst. Saf. 2018, 169, 394-402. [CrossRef] 
39. Yu, S.; Liu, J.; Yang, Q.; Pan, M. A comparison of FMEA, AFMEA and FTA. In Proceedings of the 2011 9th International Conference on Reliability, Maintainability and Safety, Guiyang, China, 12-15 June 2011; pp. 954-960.

40. Murray, G.; Edward, W.N. A Table of Integrals of the Exponential Integral. J. Res. Natl. Bur. Stand. 1969, 73, 191-210.

(C) 2019 by the authors. Licensee MDPI, Basel, Switzerland. This article is an open access article distributed under the terms and conditions of the Creative Commons Attribution (CC BY) license (http://creativecommons.org/licenses/by/4.0/). 